\title{
Diferenciación entre pobreza y exclusión: correcta identificación en un ámbito urbano intermedio
}

\author{
Differentiation Between Poverty and Exclusion: \\ Correct Identification in an Intermediate Urban Area
}

Diferenciação entre pobreza e exclusão para sua correta identificação em uma área urbana intermediaria

\author{
Cristina Calle-Espinosa ${ }^{1}$
}

Instituto de Investigaciones Económicas y Sociales del Sur, Bahía Blanca, Argentina ccalle@iiess-conicet.gob.ar

http://orcid.org/0000-0002-0543-8074 


\title{
Resumen
}

El objetivo de este artículo es reflexionar sobre las diferencias entre el concepto de pobreza y el concepto de exclusión, y así hacer una adecuada identificación de cada uno estos fenómenos en un ámbito urbano intermedio. A fin de lograr este objetivo, se presenta un marco conceptual sobre la pobreza y la exclusión, para dar lugar a un análisis en una ciudad intermedia argentina, que muestra una moderada incidencia de pobreza, pero patrones apreciables de exclusión. Posteriormente, se mide la pobreza haciendo uso de conjuntos difusos, y se evalúa la exclusión usando las dimensiones propuestas por Burchardt, Le Grand y Piachaud (1999). La principal conclusión de este artículo es que en un contexto urbano con tasas relativamente bajas de pobreza se pueden presentar situaciones de exclusión que no corresponden necesariamente a situaciones de carencia y viceversa.

Palabras clave: pobreza; exclusión social; zona urbana.

\begin{abstract}
The objective of this article is to reflect on the differences between the concept of poverty and the concept of exclusion, and thus properly identify each of these phenomena in an intermediate urban environment. In order to achieve this goal, a conceptual framework on poverty and exclusion is presented, to give rise to an analysis about an intermediate city in Argentina, which shows a moderate incidence of poverty, but appreciable patterns of exclusion. Subsequently, poverty is measured using fuzzy sets, and exclusion is evaluated using the dimensions proposed by Burchardt, Le Grand y Piachaud (1999). The main conclusion of this article is that in an urban context with relatively low poverty rates, situations of exclusion may occur that do not necessarily correspond to situations of deprivation and vice versa.
\end{abstract}

Keywords: poverty; social exclusion; urban areas.

\section{Resumo}

O objetivo deste artigo visa refletir sobre as diferenças entre o conceito de pobreza e o conceito de exclusão, buscando fazer uma adequada identificação de cada um desses fenômenos em uma área urbana intermediária. Assim sendo, apresenta-se um marco conceitual sobre pobreza e exclusão que permita a análise de uma cidade intermediária da Argentina, a qual tem uma incidência moderada de pobreza, mas com padrões importantes de exclusão. A seguir, se faz uma medição da pobreza usando conjuntos difusos e uma avaliação da exclusão utilizando dimensões propostas por Burchardt, Le Grand y Piachaud (1999). A principal conclusão deste artigo é, que num contexto urbano com taxas relativamente baixas de pobreza, podem-se apresentar situações de exclusão não necessariamente correspondentes com situações de carência e vice-versa.

Palavras-chave: pobreza, exclusão social, área urbana.

¿Cómo citar este artículo? / How to quote this article?

Calle-Espinosa, C. Diferenciación entre pobreza y exclusión para su correcta identificación en un ámbito urbano intermedio. Sociedad y Economía, (35), 78-96. https://doi.org/10.25100/sye.voi35.7296 


\section{Introducción}

La pobreza y la exclusión son fenómenos que están estrechamente ligados mediante una relación bidireccional; de forma tal que la exclusión puede privar delos recursos, a la vez que la pobreza puede excluir a los individuos de las esferas socio-económicas donde se determinan las oportunidades. Esta relación bidireccional hace que comúnmente la pobreza y la exclusión sean entendidas como un solo fenómeno y que, por lo tanto, los indicadores más utilizados no puedan distinguir entre estas dos condiciones sociales. Sin embargo, diferenciar estos esta dos puede traer ventajas tanto en el campo teórico como en el campo práctico.

Entender independientemente estas dos situaciones puede generar una mayor comprensión de cómo la pobreza se perpetúa en el tiempo, además puede permitir caracterizar situaciones de vulnerabilidad que aunque no representen per se pobreza, sí representan una imposibilidad para cubrirse frente a choques negativos de ingreso.

En cuanto al aspecto práctico, dicha diferenciación permite generar mejores indicadores capaces de identificar circunstancias que se refieren a la pobreza y/o a la exclusión, lo que permite una mejor planeación y evaluación de políticas públicas. Esto genera una mayor efectividad en cada una de las circunstancias específicas, donde no solo se logre combatir la pobreza sino también la correcta inclusión de los beneficiarios.

El objetivo de este artículo de reflexión es precisamente generar un marco conceptual que permita la diferenciación de la pobreza y la exclusión, particularmente en un ámbito urbano intermedio. Para esto se realizará, en una primera sección, una revisión de conceptos sobre estas dos problemáticas sociales, para luego presentar algunos aspectos de su medición. Con este marco se pretende señalar que las dimensiones que componen la exclusión no se deben sintetizar por medio de una adición ${ }^{2}$, tal como se hace con las mediciones de la pobreza. Esto último debido a que las dimensiones de la exclusión suelen ser independientes y de carácter dicotómico. Por lo tanto, las dimensiones de la exclusión deben ser analizadas de manera desagregada, de forma tal que sea más propicio reflexionar sobre una tipología de la exclusión, que sobre un nivel o margen, como sí es el caso de la pobreza.

Además del marco conceptual, se presenta en una segunda sección una ilustración del caso de ciudad de Bahía Blanca. Se toma como ejemplo esta ciudad intermedia argentina, ya que aunque sus indicadores de pobreza revelan una incidencia relativamente baja, la estructura de la ciudad muestrau na población excluida de la dinámica socio-económica.

Se toma como caso de estudio una ciudad intermedia, ya que esta actúa como vínculo entre las ciudades de nivel inferior y las de nivel superior, y además se relaciona con ciudades del mismo nivel jerárquico que desarrollan funciones complementarias (Preiss et al., 2012); lo que diferencia este tipo de ciudades de las dinámicas presentadas en las capitales o áreas metropolitanas ${ }^{3}$.

En el análisis de esta ciudad se presenta una medición de la pobreza mediante la aplicación de una metodología de conjuntos difusos, ya que este método permite la utilización de variables tanto categóricas como continuas. Dicha medición se acompaña de la evaluación de algunos aspectos de exclusión, diferentes a los señalados como síntomas de pobreza, sugeridos por Burchardt, Le Grand y Piachaud (1999).

2 Un ejemplo en este caso es la diferencia entre el índice multidimensional de pobreza (IMP), que agrupa sus dimensiones mediante la suma ponderada, y el at risk of poverty and/or exclusion (AROPE) que no es un indicador aditivo ya que las personas en cada dimensión se contabilizan una sola vez dentro del índice.

3 Para una mayor ilustración sobre el proceso de desarrollo urbano y sus consecuencias para la pobreza y la segregación en Bahía Blanca, leer Calle, London y Pérez (2016). 
La principal conclusión que se deriva de este artículo de reflexión es que aunque la pobreza puede ser causa y efecto de la exclusión(Gallego, 2009), pueden existir, sobretodo en contextos urbanos intermedios, situaciones de exclusión que no coinciden con situaciones de pobreza. Pero esto es observable si se tiene en cuenta que la exclusión es carácter dicotómico y no continuo como tradicionalmente se piensa la pobreza.

Aunque es posible pensar que la vulnerabilidad es una situación intermedia entre estar excluido o no, como lo expresa Castell (1997). El concepto de vulnerabilidad hace referencia particularmente a la probabilidad de que un choque resulte en una disminución de bienestar por debajo de algún umbral; por lo tanto se refiere al riesgo social o a la probabilidad de caer en pobreza (Calle et al., 2017).

Así, el individuo que se encuentra excluido experimenta una pérdida de bienestar que no está condicionado a experimentar en algún tipo de choque ${ }^{4}$. Debido a esto, es posible pensar que el concepto de vulnerabilidad requiere un enfoque de política diferente.

\section{Diferenciación entre pobreza y exclusión}

\subsection{Revisión de conceptos}

Para poder entender la diferencia entre la pobreza y la exclusión es necesario hacer un repaso de las diversas definiciones de estos dos fenómenos. En la Tabla 1 se presentan algunas de las definiciones usadas en la literatura.

Esencialmente se puede decir que el estudio de la pobreza se ha basado en diversas nociones con un enfoque cuantitativo, lo que ha permitido la identificación y la agregación del nivel

4 La principal diferencia entre el concepto de vulnerabilidad y los conceptos de exclusión y pobreza es que el primero se refiere a una situación ex ante, mientras que la pobreza y la exclusión son términos ex post. de bienestar de las personas que se consideran pobres (Feres y Mancero, 2001).

Mientras que el término exclusión partió de la concepción cualitativa de inclusión social, como un proceso de integración en las dimensiones de orden económico, político y social. Este concepto se centra en la participación efectiva en el proceso de toma de decisiones, el estatus productivo y el rol de los lazos sociales (Laparra et al., 2007).

Sin embargo, son muchos los puntos de encuentro entre la pobreza y la exclusión, por lo tanto, en literatura se han desarrollado dos líneas teóricas diferentes. La primera línea concibe la exclusión solamente como un componente de la pobreza, mientras que la segunda línea define la exclusión como un fenómeno independiente, que abarca muchos más aspectos que los considerados en la pobreza (Bradshaw et al., 2000).

En lo que respecta a la primera línea, se encuentran autores como Spicker (2007), quien establece que la exclusión es una dimensión de la pobreza en su acepción más amplia. Esta acepción se basa en la posición social, y se evidencia por la falta de derechos para disfrutar los bienes y servicios, y así gozar plenamente de la sociedad.

En ese sentido, cuando el concepto de pobreza es definido en su vertiente más amplia, los conceptos de exclusión y desigualdad están integrados en él. Por lo tanto, las mediciones de la pobreza incorporan la exclusión siempre y cuando se incluyan aspectos no monetarios relacionados, entre otras cosas, con el capital social y las relaciones de los individuos en la comunidad (Arriagada, 2005) 5 .

5 El enfoque de este artículo no pretende ahondar en el estudio de la desigualdad y de su principal medida, el índice GINI. Igualmente, existe una diferenciación entre capital social y exclusión social que es presentada por Daly y Silver (2008), pero al no ser el objeto del trabajo no se profundiza en dicha diferencia. 
Tabla 1. Definiciones de pobreza y exclusión

La pobreza puede ser definida como un concepto material, donde una persona es pobre porque no tiene algo que necesita. También como una circunstancia económica donde se tiene un menor estándar de vida con respecto a la sociedad. O se define como una circunstancia social, donde se es pobre si se pertenece a una clase inferior (Spicker, 2007).
Un individuo es socialmente excluido si él o ella reside en una sociedad, pero por alguna razón más allá de su control no puede participar en las actividades normales de la ciudadanía en esa sociedad, aunque le gustaría hacerlo (Burchardt et al., 1999).
La pobreza como la carencia humana derivada de las limitaciones de las fuentes de bienestar, el tiempo y el acceso a los servicios gubernamentales gratuitos (Boltvinik, 2003).

Situación donde los individuos no poseen bienes y servicios requeridos para vivir. $\mathrm{O}$ una situación donde el individuo vive con menos que otras personas (Feres y Mancero, 2001).
La incapacidad de participar efectivamente en la vida económica, social, cultural y política; también la alienación y distancia de la sociedad predominante (Duffy, citado en Levitas, 2006).
Es un proceso social de pérdida de integración que incluye no solo la falta de ingresos y el alejamiento del mercado de trabajo, sino también un descenso de la participación social y por tanto una pérdida de derechos sociales (Laparra et al., 2007).
Un síndrome situacional en el que se asocian el infraconsumo, las precarias condiciones de vivienda, los bajos niveles de educación, las malas condiciones sanitarias, la inserción inestable al aparato productivo, y poca participación (Arriagada, 2005).
Negación o no realización de los derechos sociales y políticos de ciudadanía (Romm, citado en Klasen, 1998).
Pobreza como falta de desarrollo de capacidades, como negación de la democracia, la falta de bienestar y autonomía y la negación de oportunidades (Gallego, 2009).
Rupturas sucesivas que, arrancando del corazón de la economía, la política y la sociedad, van alejando a personas, grupos, comunidades y territorios con respecto a los centros de poder, los recursos y los valores dominantes (Estivill, citado en Raya-Díez, 2007).
La pobreza puede ser definida en múltiples espacios, desde los aspectos materiales de la vida a los aspectos sociales y culturales. En términos de utilidad o en términos de libertad (Laderchi, Saith y Stewart, 2003).
La exclusión social implica centrarse en los procesos y las relaciones que causan la privación (De Haan, 2000).

Fuente: recopilación y traducción propia.

Gallego Duque (2009) también reconoce la relación que existe entre la pobreza y la exclusión, ya que ambos son problemas complejos y multicausales. En este aspecto, la autora subraya que el enfoque de capacidades de Sen permite entender tanto la problemática de la pobreza como la problemática de la exclusión; sin embargo, es necesario tener presente las diferencias entre estos conceptos. En una cita a Sen expresa:
En primer lugar tenemos buenas razones para valorar no ser excluidos de las relaciones sociales, en este sentido la exclusión social puede ser directamente parte de la pobreza de capacidades [...]. En segundo lugar, estando excluido de las relaciones sociales se puede conducir a otras formas de privación y también de ese modo, limitar nuestras oportunidades de vida (Sen, citado en Gallego, 2009, p. 11). 
Dado lo anterior, es posible pensar que la exclusión en estos términos va más allá de la falta de participación de los beneficios económicos o de la ruptura de los vínculos sociales y políticos de un individuo, pero que se puede entender como una falla de un funcionamiento, más específicamente como privación en la capacidad de sociabilización. Por lo tanto, la exclusión está ligada a la pobreza ${ }^{6}$.

En resumen, se puede decir que la definición de la pobreza ha evolucionado para abarcar además de aspectos económicos, aspectos políticos y sociales, haciendo que el concepto de pobreza sea bastante próximo al de exclusión. Sin embargo, el término de exclusión se ha preferido a circunstancias donde existe un Estado de bienestar que promueva un ingreso mínimo y el acceso a los bienes y servicios básicos (Bhalla y Lapeyre, 1997).

En cuanto a la segunda línea teórica, que distingue entre pobreza y exclusión, se encuentran autores como: Navarro y Larrubia (2006) y Hernández Pedreño (2010), quienes señalan que mientras la pobreza es unidimensional (carencia económica), la exclusión es multidimensional, ya que incluye aspectos políticos y sociales. Sin embargo, este argumento habría que revisarse de cara al actual desarrollo teórico de la pobreza multidimensional como los presentados por Alkire y Santos (2010) y Boltvinik (2013)7.

6 Pero esta concepción ignora que el funcionamiento puede fallar, ya sea por una falta de desarrollo, por una falta de recursos o por la exclusión en sí misma. Así como en una persona paralítica, el funcionamiento de desplazarse libremente puede fallar porque no se dispone de una silla de ruedas, por la falta de dinero para adquirir una silla de ruedas o porque no existen rampas. Aunque eliminar la exclusión puede significar un desarrollo social, esto no necesariamente implica una mejora en cuestión de pobreza, tal y como es el caso de la instalación de rampas en lugares pobres donde los individuos no cuentan con sillas de ruedas.

7 Es importante indagar si la conceptualización de la pobreza multidimensional realmente toma en cuenta aspectos de las dimensiones sociales y políticas, o solo se limita a una restructuración de la dimensión económica.
Por otro lado, algunos autores como Touraine (1991) rechazan la vinculación de la exclusión con el concepto de pobreza, argumentando que la pobreza se refiere a la posición de las personas en un eje vertical de estatus socio-económico, mientras que la exclusión tiene una forma geométrica diferente; es decir, la exclusión se refiere a estar fuera o dentro del círculo social. Esta diferenciación también la hizo la Comisión Europea señalando que el reto de los gobiernos y los responsables de la política social ha cambiado de naturaleza, al tratarse ahora de un mecanismo que excluye a partes de la población de los beneficios económicos y sociales, y del progreso en general.

(...) Todos los debates enfatizan el carácter estructural de un fenómeno que tiende a establecer dentro de la sociedad un mecanismo que excluye parte de la población de la vida económica y social, y de su parte de la prosperidad general (...) El problema ahora no es sólo una disparidad entre la parte inferior y superior de la escala social (arriba/abajo) sino también entre aquellos cómodamente situados dentro de la sociedad y los marginados (adentro y afuera) (Commission Communities of European, 1992, p. 7$)^{8}$.

Burchardt, Le Grand y Piachaud (1999) también hacen un tratamiento dicotómico de la exclusión, donde los indicadores señalan si el individuo está o no excluido en alguna dimensión. Para estos autores es absurdo establecer categorías continuas para dimensiones como el aislamiento social o la desvinculación política; mientras que dimensiones como el ingreso, puede ser dividida en aquellos que están sobre algún umbral y aquellos que están por debajo.

Además es posible pensar que la exclusión, a diferencia de la pobreza, tiene una fuerte dimensión territorial ${ }^{9}$, más precisamente está li-

8 Traducción propia.

9 Aunque la pobreza también posee una connotación espacial, es posible rastrear el origen de la exclusión con el incremento de la tasa de urbanización y el surgimiento de áreas con menos dotación de bienes y servicios públicos próximas a áreas que poseían todos los benefi- 
gada a la formación y expansión de las ciudades. Normalmente, la exclusión en las urbesse expresa como la dicotomía centro-periferia, donde el fenómeno de exclusión social está acompañado de la no asimilación cultural (Touraine, 1991).

Subirats (2005) muestra que es mucho más común encontrar en la ciudad procesos de exclusión social y segregación territorial. La exclusión social en ámbitos urbanos está caracterizada por la fragmentación de la sociedad, la precarización del mercado laboral y el déficit de inclusión del estado de bienestar.

Esto hace que la exclusión que se presenta en las ciudades sea diferente a otros problemas sociales presentados en otros ámbitos territoriales como por ejemplo el campo, donde se pueden presentar no solo magnitudes de pobreza considerables ${ }^{10}$, sino también procesos de exclusión de los recursos productivos, lo que condiciona negativamente el bienestar del individuo rural.

Sin embargo, el enfoque en el que se fundamenta este trabajo se centra en la ciudad, ya que es allí donde confluyen oportunidades económicas, políticas y sociales propias de los procesos de aglomeración. A pesar de eso, existen grupos que no pueden acceder a esas oportunidades; esto tiene características diferentes a la mera privación y aporta rasgos importantes al desempeño urbano.

\subsection{Algunas consideraciones sobre las mediciones}

Una vez hecho un repaso sobre los conceptos de pobreza y exclusión, así como también de las principales características que diferencian a ambos términos, se procede a presentar algunas consideraciones metodológicas sobre la forma como tradicionalmente se han medido estos dos problemas. Para finalmente dimensionar

cios socio-económicos de la aglomeración.

10 La CEPAL y la FAO estimaron que en el 2010 la pobreza e indigencia rural alcanzaron $53 \%$ y $30 \%$ respectivamente en Latinoamérica y el Caribe (Faiguenbaum, 2013). ambos fenómenos en el contexto de una ciudad de tamaño intermedio.

Se puede decir, que desde el principio del siglo pasado, en la agenda política ha existido una fuerte preponderancia por vigilar las condiciones de pobreza", lo cual llevó a un inmenso desarrollo de indicadores sobre este tema. Sin embargo, la investigación sobre la exclusión solamente se dinamizó a partir de la década de 1980 , sobre todo en los países europeos ${ }^{12}$.

Debido a las múltiples definiciones de pobreza anteriormente señaladas, se ha desarrollado una variedad de mediciones, a partir de las cuales han surgido algunos debates, como por ejemplo el debate entre los indicadores relativos o absolutos; entre los enfoques directos e indirectos; y más recientemente, entre la perspectiva objetiva y la perspectiva subjetiva (Peralta, García y Johnson, 2006).

También se ha debatido entre la pobreza como un fenómeno ligado a una dimensión (ingreso principalmente) o como un problema multidimensional. Por un lado se ha argumentado que existe una alta correlación entre el ingreso y otras dimensiones no monetarias $\mathrm{y}$, por lo tanto, cuantificar solamente el ingreso es una medida cercana al estado de pobreza.

Por otro lado, se ha argumentado que existen indicadores no monetarios, como la educación y la salud o el tiempo libre, que deben incluirse al momento de cuantificar el nivel de vida. Es precisamente definir qué se entiende por nivel de vida el punto clave en la medición de pobreza.

Una vez hecha esta precisión, se determina un espacio en el cual se pueda hacer un

11 Atkinson(1987) señala el trabajo de Sir Arthur Bowley de 1915 como pionero en la medición de la pobreza. Aunque hay trabajos previos por parte de Luis Vives con el tratado De subventione pauperum en 1524 , estos no representan una metodología de medición.

12 Más específicamente, el interés sobre la exclusión social se dinamizó a partir del II Programa de Lucha contra la Pobreza y la Exclusión Social de la Comisión Europea en 1989. 
ordenamiento de mayor a menor. La mayoría de los estudios sobre la pobreza se han centrado en tres aspectos: la satisfacción de las necesidades, la cantidad de recursos disponibles y los estándares de vida (Feres y Mancero, 2001). El estándar de vida a su vez puede ser definido como el grado de utilidad que brinda el consumo de bienes y servicios, o como el conjunto de capacidades que tiene un individuo. Sin embargo, la mayoría del análisis de la pobreza está basado en un enfoque utilitarista (Domínguez y Martín, 2006).

Domínguez Domínguez y Martín Caraballo (2006) presentan una recopilación de las principales cuestiones metodológicas acerca de la medición de pobreza. En dicha revisión se incluye el cálculo de la línea de pobreza y de las escalas de equivalencia. También presentanel enfoque axiomático, propuesto inicialmente por Sen, y las medidas de pobreza basadas en el enfoque de bienestar ${ }^{13}$.

En cuanto a las medidas de exclusión social se puede decir que el desarrollo metodológico no ha sido tan extenso como el de las mediciones de pobreza, debido entre otras cosas a su reciente desarrollo teórico, y a una serie de problemas metodológicos que aparecen a la hora de elaborar un indicador para la exclusión.

Bhalla y Lapeyre (1997) apuntan que agregar indicadores económicos, sociales y políticos puede carecer de sentido, especialmente cuando estos tres aspectos de la exclusión no necesariamente se mueven en la misma dirección. Además, también es posible que los ponderadores de cada una de las dimensiones varíen según

13 Para una ilustración más profunda acerca de la medición de la pobreza por ingreso, remitirse a Foster, Greer y Thorbecke (1984) que presentan un índice de pobreza que además de cumplir con el axioma de monotonicidad, y de sensibilidad a las transferencias, es aditivamente descomponible en subgrupos. También Atkinson (1987) que analiza la comparabilidad entre diferentes líneas de pobreza mediante la condición de dominancia estocástica. Además, Foster (1998) hace una presentación de consideraciones sobre el carácter absoluto o relativo en la medición de pobreza, tanto para la etapa de identificación como para la etapa de agregación. los diferentes estados del desarrollo de un determinado país.

Por ejemplo, en países de ingreso bajo puede ser más apropiado concederle un gran peso a la dimensión económica, y medir la exclusión mediante indicadores que den cuenta y razón de la profundidad de la pobreza y la desigualdad del ingreso ${ }^{14}$. Mientras que, por otro lado, en sociedades con un ingreso superior se debería dar mayor peso a indicadores de la dimensión social y política.

Particularmente, la dimensión social se refiere al acceso a los bienes y servicios públicos ${ }^{15}$, así como al acceso al mercado de trabajo, sobre todo el acceso al empleo decente y no precarizado. Igualmente se refiere a la participación social, como son la participación en sindicatos y la participación en asociaciones locales y agremiaciones.

En cuanto a la dimensión política, la medición de la exclusión también debe contemplar el grado de libertad política, que incluye la seguridad personal, el Estado de derecho, la libertad de expresión, la participación política y la igualdad de oportunidades. Es importante señalar que se ha encontrado una correlación positiva entre la libertad política y el nivel de ingreso, así como también entre los índices de desarrollo humano y los índices de desarrollo político (Sen, 2000).

Por lo anterior, se podría pensar que para la evaluación de las políticas contra la exclusión puede ser más útil el uso de un conjunto de indicadores que el uso de un solo índice compuesto. Pero a pesar de la dificultad de encontrar una sola medida de exclusión adecuada que pueda reflejar además de la dimensión económica, las dimensiones social y política, se han

14 Bhalla y Lapeyre (1997) sugieren la utilización del indicador propuesto por Foster, Greer y Thorbecke (1984), ya que logra identificar la distribución de los hogares acorde a diferentes grados de pobreza.

15 Los cuales se ven reflejados en indicadores de expectativa de vida, mortalidad infantil, analfabetismo y escolaridad, entre otros. Algunos de ellos también presentes en las mediciones de pobreza multidimensional. 
desarrollado índices de exclusión compuestos, basados en niveles de privación.

Dos ejemplos de este tipo de medición son los propuestos por Chakravarty y D'Ambrosio (2006) y Bossert, D’ambrosio y Peragine (2007). Estos indicadores se basan en el concepto de funcionamiento, que se desprende de los planteamientos de Sen y que está en concordancia con las medidas multidimensionales de pobreza que se han desarrollado ${ }^{16}$.

Estas medidas también presentan un enfoque axiomático, donde la exclusión social es una función real evaluada en la medida de privación de los individuos ${ }^{17}$. Estas medidas son separables en subgrupos, y cumplen algunos criterios como la normalidad y marginalidad no decreciente, esto último implica un aumento en la exclusión social cuando la medida de privación de un individuo es incrementada en una unidad ${ }^{18}$.

Una de las características principales de este tipo de indicadores es que se pueden aplicar los criterios de dominancia, particularmente el criterio de dominancia de Lorenz, que también es usado en las medidas de pobreza. Sin embargo, este tipo de medidas entienden la exclusión como un estado de privación dinámica, por lo tanto, la diferencia entre privación y exclusión en este caso resulta ser el desarrollo en el tiempo.

En otro tipo de enfoque, Raya-Díez (2007) elabora una herramienta que permita la medición y diagnóstico de los procesos de exclusión social, con un planteamiento que no solo busca determinar un grado de intensidad de la situación de

16 Es importante notar que a diferencia de las medidas multidimensionales de pobreza, las medidas de exclusión no se centran el medir un déficit a partir de un umbral sino que se centran en la imposibilidad de participar; por lo tanto, permiten el uso de funcionamientos cuantitativos y funcionamientos cualitativos.

17 En este caso la medida de privación es el número de funcionamientos del cual una persona es excluida en el tiempo; este razonamiento exige que exista un ordenamiento de funcionamientos en cuanto a su relevancia.

18 Adicionalmente Chakravarty y D'Ambrosio (2006) también cumplen con el axioma de monotonicidad. exclusión, sino también en qué ámbitos vitales se está afectando en mayor medida. Esto es una alternativa viable frente a las mediciones de exclusión axiomáticas.

Finalmente, Navarro y Larrubia (2006) hacen una recopilación de los indicadores de exclusión más utilizados por la OCDE y la ONU. También elaboran un sistema de indicadores de exclusión con una perspectiva territorial y urbana microespacial. La novedad de esta propuesta es la utilización de un sistema de información georreferenciada.

\section{Diferenciación entre pobreza y exclusión en Bahía Blanca}

\subsection{Características de la ciudad de Bahía Blanca}

Bahía Blanca es una ciudad argentina intermedia, cuyas dinámicas económicas y demográficas se diferencian de otras ciudades de Argentina debido, entre otras cosas, a su ubicación en el sur de la provincia de Buenos Aires, lo que la convierte en un eje articulador de dicha región.

Esta ciudad tiene un total poblacional estimado en 300.000 habitantes según el censo del 2010. Según el Centro Regional de Estudios Económicos de Bahía Blanca, en el 2014 su producto bruto constituyó un 0,8\% al PIB de Argentina (Centro Regional de Estudios Económicos de Bahía Blanca Argentina, 2014a).

Se estima que la producción de la industria manufacturera representa un $33,2 \%$ del producto bruto de la ciudad, mientras que el comercio contribuye con $17,6 \%$. La mayor parte de la actividad en estos dos sectores se encuentra concentrada en un polo petroquímico y en un puerto marítimo, que comercia principalmente granos. De hecho, para el 2014, el valor agregado bruto generado por la refinación de petróleo, químicos y plásticos constituía un $24 \%$ del ingreso total generado en la ciudad de Bahía 
Blanca (Centro Regional de Estudios Económicos de Bahía Blanca Argentina, 2014a).

En cuanto a los indicadores de pobreza, según el INDEC, en el 2013 las personas por debajo de la línea de pobreza, medida mediante la canasta básica total, representaban $8,8 \%$ de la población total. Mientras que las personas por debajo de la canasta básica de alimentos, o sea en condición de indigencia, representaban un $3,2 \%$. Los indicadores de pobreza y de indigencia con respecto al número de hogares eran de $5,6 \%$ y $3,2 \%$ respectivamente.

Una estimación paralela a la anterior, realizada por el Centro de Estudios Económicos de Bahía Blanca, Argentina, sugiere que en el 2013 el porcentaje de pobreza era de $29,3 \%$; mientras que $6 \%$ de la población se encontraba por debajo de la línea de indigencia. En tanto la proporción de hogares pobres era de $18,3 \%$, y la proporción de hogares indigentes era de 3,6\% (Centro Regional de Estudios Económicos de Bahía Blanca Argentina, 2014b).

Por otra parte una medida de pobreza, según el índice de necesidades básicas insatisfechas, con información recolectada del Censo Nacional del 2010, reporta que 3,7\% de los hogares de la ciudad reportan al menos una necesidad básica insatisfecha, siendo el hacinamiento crítico la necesidad insatisfecha más representativa, con una incidencia de $2,1 \%$.

En una estimación más reciente Santos (2016) determina que en el 2015 un 3\% de la población es indigente, mientras que $8 \%$ de la población es pobre ${ }^{19}$. Lo que es para los dos casos una cifra menor que para el total del país.

En cuanto a la exclusión es más difícil encontrar un indicador preciso que dé cuenta y razón de este fenómeno particularmente para Bahía Blanca, sin embargo se puede hacer referencia a las cifras del mercado laboral como una

19 Esta estimación se hace mediante le estimación de una canasta básica local.

sociedad y economía No. 35, 2018 • pp. 78-96 aproximación a las consideraciones de exclusión en la ciudad.

En cuanto a este tema se puede decir que la tasa de actividad de la ciudad en promedio entre 2014 y 2015 fue $46,26 \%$, cifra un poco mayor a la de todo el país. Por otro lado, la tasa de desempleo de la ciudad entre 2014 y 2015 presenta en promedio $7,66 \%$ de la población económicamente activa, cifra similar al comportamiento nacional (Centro Regional de Estudios Económicos de Bahía Blanca Argentina, 2017).

\subsection{Medición de pobreza y exclusión en Bahía Blanca}

\subsubsection{Pobreza en Bahía Blanca}

La medición de la pobreza para este trabajo se hace por medio de la aplicación de una línea de pobreza de conjuntos difusos. Se toma esta medida, en pos de la idea de que la pobreza se puede analizar mediante variables continuas y categóricas, donde la condición de pobreza es determinada de manera gradual. Se sigue la metodología propuesta por Morales-Ramos y Morales-Ramos (2008).

Según esta metodología, el nivel de pobreza se determina mediante una función de membresía o pertenencia $\mu_{A}(i)$, que es un conjunto difuso A, donde existe un conjunto de $\mathrm{K}$ indicadores sintomáticos de pobreza $\left(Y_{1}, Y_{2}, \ldots, Y_{K}\right)$. Siendo $Y_{i j}$ el indicador $j$ para el individuo $i$. Estos indicadores sintomáticos de pobreza pueden tomar forma continua o categórica.

Así $\mu_{A}$ (i) es un mapeo de un conjunto en un intervalo $[0,1]$ que determina el grado de pertenencia de un individuo al conjunto de pobres A. Ahora si $y_{j}^{\prime}$ es el máximo valor de $Y_{j}$ para el cual el individuo es considerado pobre; y $y_{j}^{\prime \prime}$ es el valor de $Y_{j}$ para el cual el individuo deja de ser pobre $^{20}$. Entonces:

20 Note que esta metodología puede generalizarse para línea de pobreza tradicional cuando $y_{j}^{\prime}=y_{j}^{\prime \prime}$ 


$$
\mu_{A}(i)=\left\{\begin{array}{c}
1 \text { Si } 0 \leq y_{i j} \leq y_{j}^{\prime} \\
0<\mu_{A}<1 \text { Si } y_{j}^{\prime}<y_{i j} \leq y_{j}^{\prime \prime} \\
0 \text { Si } y_{i j}>y_{j}^{\prime \prime}
\end{array}\right\}
$$

Una de las formas que puede tomar la función de membresía es $\mu_{A}(i)=\frac{y_{j}^{\prime \prime}-y_{i j}}{y_{j}^{\prime \prime}-y_{j}^{\prime}}$.

El grado de pobreza de cada individuo será la sumatoria de su nivel de carencia en cada uno de los indicadores sintomáticos de pobreza ${ }^{21}$

$$
U_{i}=\frac{1}{k} \sum_{i=1}^{k} \mu_{A}(i) \quad \text { que toma valores entre o y } 1
$$

El índice difuso de pobreza es la sumatoria de cada una de las funciones de pertenencia para todos los individuos, así:

$$
P=\frac{1}{n} \sum_{i=1}^{n} U_{i}
$$

Los indicadores sintomáticos de pobreza utilizados en este caso se pueden apreciar en el Anexo 1. Dichos indicadores son extraídos de la Encuesta Permanente de Hogares (EPH) 2015 que presenta una muestra de 1409 individuos. En este ejercicio se toman en cuenta la dimensión educativa, la calidad de la vivienda y el ingreso. La proporción de afectados según el valor de la función de pertenencia se puede ver en la Gráfica 1.

Usando esta metodología se puede establecer que $18,13 \%$ de la población de la ciudad puede considerarse pobre, sin embargo como se puede ver en la gráfica 1 los valores que toma la función de pertenencia al conjunto de pobreza son inferiores a 0,8 , lo que significa que ningún individuo de la encuesta presenta carencias en todos los indicadores de las tres dimensiones tenidas en cuenta.

21 La función de pertenencia de la pobreza depende de la importancia que tiene cada indicador. En este caso se toma cada indicador con el mismo peso relativo, ya que determinar un valor diferente para cada indicador requiere un análisis moral que supera los alcances del trabajo.
En la gráfica 1 se dimensiona la exclusión con el fin de hacer una comparación con la anterior medida de pobreza.

\subsubsection{Exclusión en Bahía Blanca}

El análisis de la exclusión en este caso sigue la metodología presentada por Burchardt et al. (1999), la cual postula que para evaluar la exclusión es necesario tener en cuenta cinco dimensiones: el consumo, la riqueza, la actividad productiva, la actividad política y la actividad social. Estas dimensiones representan para ese autor la participación en actividades fundamentales.

A partir nuevamente de la EPH 2015 se toman los indicadores de cada dimensión y se presentan en la Tabla 222; estos indicadores, a excepción del ingreso, son diferentes a los utilizados para medir la pobre$\mathrm{za}$, ya que se basan en la idea de la exclusión como un fenómeno dicotómico.

Como la dimensión de consumo es medida mediante el ingreso, más específicamente mediante el mismo indicador que en el análisis de la pobreza hecho anteriormente, el comportamiento es el mismo. Por otro lado, la dimensión de riqueza que evalúa el porcentaje de individuos que no son propietarios de su vivienda o que no pertenecen a un régimen jubilatorio muestra un valor alto, ya que en esa dimensión solo 2,83\% está excluido en todos los indicadores de esa dimensión.

En la dimensión de productividad también son cifras bajas, ya que el desempleo es de $3,34 \%$, y de los ocupados solo 6,33\% reportan subempleo. Finalmente, en la dimensión social que se refiere a la vinculación a la protección social, particularmente si no cuenta con cobertura de salud de cualquier clase, el porcentaje es de $19,73 \%$. El 70,26\% de la población se ve excluido en al menos un indicador de las cuatro dimensiones.

22 Sin embargo, dada la información de la Encuesta Permanente de Hogares fue imposible obtener información sobre la dimensión política, y la dimensión social fue sustituida por la cobertura al sistema de salud pública. 


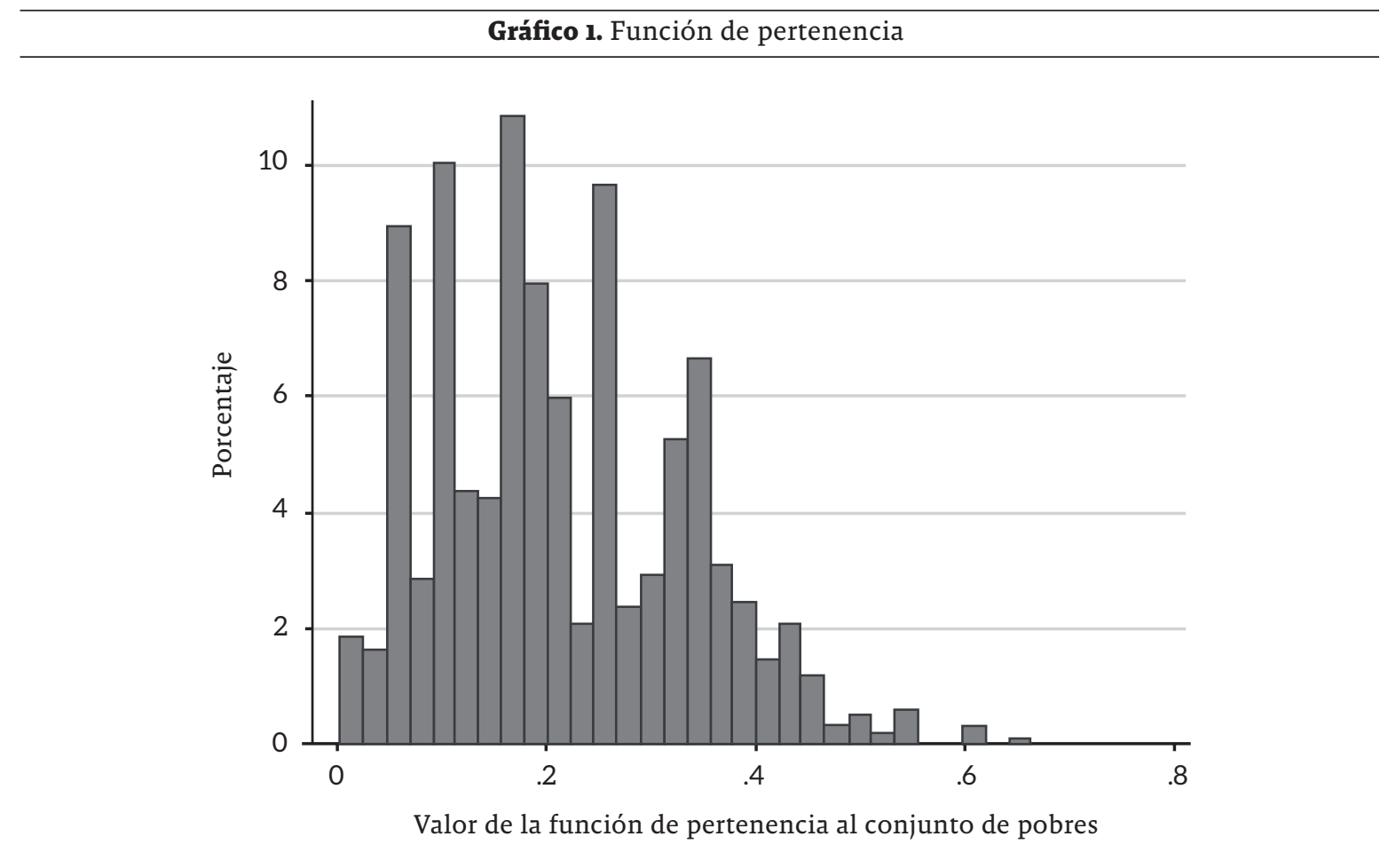

Fuente: elaboración propia.

Tabla 2. Indicadores para medir la exclusión

\begin{tabular}{ccc}
\hline & Variable & \\
\hline & Ingreso inferior al 60\% de la media* & $31,72 \%$ \\
\hline & No propietario de la vivienda & $29,88 \%$ \\
\hline & No cuenta con aportes a pensión & $7,3 \%$ \\
\cline { 2 - 3 } & Desempleo & $3,34 \%$ \\
\hline
\end{tabular}

* Se elige el 60\% de ingreso medio como la línea de pobreza relativa elegida para la Unión Europea.

Fuente: elaboración propia.

La Tabla 3 muestra la matriz de intercepción en cada una de los indicadores de pobreza y la exclusión. Como se puede ver la relación entre las dimensiones de pobreza y exclusión no es evidente, exceptuando el caso de los hogares con un piso de mala calidad que presenta el $55,9 \%$ de hogares que también son excluidos en el consumo. En el resto de los casos no se puede 
apreciar que los indicadores de pobreza tengan un grado considerable de correspondencia con los indicadores de exclusión.

Sobre todo en el caso del analfabetismo donde a pesar de cumplirse el indicador de pobre$\mathrm{za}$, hay baja incidencia en los indicadores de exclusión, especialmente en el desempleo, el subempleo y los aportes a pensión; esto puede estar explicado por el hecho de que la mayoría de población adulta analfabeta es de la tercera edad, y por lo tanto no se encuentra dentro de la población que participa activa.

En contraposición, se encuentran los indicadores de pobreza que tienen en cuenta la calidad física de la vivienda; estos indicadores presentan mayor concordancia con los indicadores de exclusión, sobre todo con la dimensión de consumo, riqueza y participación social. Especialmente el indicador de la calidad del piso de la vivienda, donde $40 \%$ son excluidos de la tenencia formal de la vivienda, y $44,1 \%$ son excluidos de la cobertura médica.

Existe una menor relación en los indicadores de exclusión que tienen que ver con la actividad productiva. Por ejemplo, solo $3,7 \%$ de las personas que reportan desempleo pertenecen a una vivienda con techos de mala calidad. Igualmente, solo $2,2 \%$ de los empleados que están subempleados tienen niveles bajos de educación.

Tabla 3. Indicadores de exclusión versus indicadores de pobreza

\begin{tabular}{|c|c|c|c|c|c|c|c|c|c|c|c|c|}
\hline \multirow[t]{2}{*}{ Pobreza } & \multicolumn{2}{|c|}{$\begin{array}{c}\text { Ingresos } \\
\text { por debajo } \\
\text { de } 60 \% \text { de la } \\
\text { media }\end{array}$} & \multicolumn{2}{|c|}{$\begin{array}{l}\text { No tenencia de } \\
\text { vivienda }\end{array}$} & \multicolumn{2}{|c|}{$\begin{array}{c}\text { Sin aportes a } \\
\text { pensión }\end{array}$} & \multicolumn{2}{|c|}{ Desempleo } & \multicolumn{2}{|c|}{ Subempleo } & \multicolumn{2}{|c|}{$\begin{array}{l}\text { Sin cobertura } \\
\text { en salud }\end{array}$} \\
\hline & No & $\mathrm{Si}$ & No & $\mathrm{Si}$ & No & $\mathrm{Si}$ & No & $\mathrm{Si}$ & No & $\mathrm{Si}$ & No & $\mathrm{Si}$ \\
\hline & $100 \%$ & $100 \%$ & $69,8 \%$ & $30,2 \%$ & $89,7 \%$ & $10,1 \%$ & $95 \%$ & $4,9 \%$ & $96,1 \%$ & $3,8 \%$ & $63,5 \%$ & $36,4 \%$ \\
\hline & $75 \%$ & $25 \%$ & $87,5 \%$ & $12,50 \%$ & $100 \%$ & $0 \%$ & $100 \%$ & $0 \%$ & $100 \%$ & $0 \%$ & $87,5 \%$ & $12,5 \%$ \\
\hline & $69,1 \%$ & $30,9 \%$ & $77 \%$ & $23 \%$ & $86,9 \%$ & $13,1 \%$ & $94,4 \%$ & $5,6 \%$ & $97,8 \%$ & $2,2 \%$ & $74,3 \%$ & $25,7 \%$ \\
\hline & $44,1 \%$ & $55,9 \%$ & $60 \%$ & $40 \%$ & $86 \%$ & $14 \%$ & $93 \%$ & $7 \%$ & $97,6 \%$ & $2,4 \%$ & $55,9 \%$ & $44,1 \%$ \\
\hline & $65 \%$ & $35 \%$ & $81,2 \%$ & $18,8 \%$ & $91,7 \%$ & $8,3 \%$ & $96,3 \%$ & $3,7 \%$ & $97,2 \%$ & $2,8 \%$ & $79,7 \%$ & $20,3 \%$ \\
\hline & $51,4 \%$ & $48,6 \%$ & $83,6 \%$ & $16,4 \%$ & $90,5 \%$ & $9,5 \%$ & $95,7 \%$ & $4,2 \%$ & $96,7 \%$ & $3,3 \%$ & $88,8 \%$ & $28,8 \%$ \\
\hline
\end{tabular}

Fuente: elaboración propia. 


\section{Conclusiones}

En las sociedades es común apreciar simultáneamente situaciones de pobreza y exclusión, y por lo tanto es común confundir estos dos fenómenos, tanto en la práctica como en el desarrollo teórico de las ciencias sociales. Sin embargo, han sido muchos los aportes que han señalado las ventajas de diferenciar los dos conceptos, y desarrollar herramientas independientes para analizar la evolución de cada uno de los problemas.

No obstante, esta tarea no ha sido fácil debido a la gran variedad de formas como las ciencias sociales han definido la pobreza y la exclusión. A lo largo del desarrollo teórico de estos dos conceptos han existido puntos de encuentro, donde los términos pueden ser entendidos como sinónimos. Pero también han existido marcadas diferencias, sobre todo en la relevancia que le han dado los organismos internacionales a la hora de diseñar los objetivos de política de bienestar.

De la revisión de la literatura se puede extraer que existen dos líneas teóricas en cuanto al análisis de estos dos problemas sociales. Por un lado existen aquellos que opinan que la exclusión es solo una dimensión de la pobreza y, por lo tanto, cuando este fenómeno es definido en su acepción más amplia, las mediciones de pobreza incluyen los aspectos más relevantes de la exclusión. La segunda línea especifica que la exclusión y la pobreza se manifiestan en la sociedad de manera diferente y por lo tanto se deben aplicar mediciones diferenciadas.

En el primer enfoque se encuentran las teorías que entienden la pobreza como un fenómeno multidimensional y que, por lo tanto, otros conceptos como la desigualdad y la exclusión pueden ser subordinados al estudio de la pobreza. En el segundo enfoque se encuentran las teorías que entienden la pobreza como un fenómeno que se limita a aspectos económicos y que, por lo tanto, no puede dar cuenta de los aspectos sociales o políticos que conciernen a la exclusión.
Otra diferencia que se ha señalado entre la pobreza y la exclusión tiene que ver con el alcance territorial de los dos conceptos. Mientras que, por un lado, la exclusión se enmarca la mayoría de las veces a territorios urbanos, por otro lado, la pobreza como fenómeno social no se ha restringido a ningún ámbito territorial, existiendo tanto pobreza urbana como pobreza rural.

La exclusión se ha relacionado principalmente a las ciudades, donde existen áreas con bajas tasas de desarrollo y acceso a bienes y servicios públicos, próximas a áreas que aprovechan todos los beneficios de la aglomeración. Por otro lado, la exclusión en contextos rurales, se refiere a la participación y el derecho de recursos productivos que determinan el desempeño socio-económico del individuo, pero que no están relacionadas con las ventajas de la aglomeración.

Particularmente, la pobreza se ha medido por medio de la cuantificación del nivel de vida. Dicho nivel que ha tomado la connotación de la satisfacción de las necesidades, de la cantidad de recursos disponibles, o de los estándares de vida. A su vez el estándar de vida puede ser entendido como la utilidad que se deriva del consumo de bienes y servicios o como el conjunto de capacidades con las que cuenta un individuo.

Por consiguiente, la medición de la pobreza, generalmente, se basa en determinar un umbral en algún punto de dicha categoría continua, donde por debajo de dicho límite se encuentren los individuos pobres. Mientras que los niveles de vida superiores a dicha división no correspondan a situaciones de pobreza. De esta frontera se desprende la medición de la incidencia de la pobreza y la brecha de la pobreza.

La exclusión, por otro lado, se ha medido mediante una serie de dimensiones categóricas, que además del nivel de vida del individuo, también incluye condiciones sociales y políticas. Las características de estas dimensiones imposibilitan la agregación de la exclusión en un solo indicador. Por lo tanto, de las medidas 
de exclusión se puede desprender una tipología de exclusión, más que una brecha de exclusión.

Particularmente, en un ámbito urbano de tamaño intermedio, como la ciudad argentina de Bahía Blanca, se ve una baja incidencia de pobreza, pero mayores patrones de exclusión. Así, a pesar de que existe una relativa baja incidencia de pobreza, alrededor de $18,13 \%$, el $70,26 \%$ de la población está excluida en al menos un indicador dentro de las dimensiones de consumo, riqueza, productividad y en la dimensión social.

Igualmente, en esta ciudad no hay evidencia concluyente de la confluencia de estos dos fenómenos, sobretodo en la circunstancias de exclusión, cuando esta es medida mediante el desempleo, donde solo $3,7 \%$ de los individuos desempleados pertenecen a hogares con techo de mala calidad; y mediante el subempleo, donde solo $2,2 \%$ son considerados pobres por baja educación.

Sin embargo, la relación entre los dos fenómenos es más evidente cuando se tienen en cuenta otros indicadores de pobreza. Por ejemplo, el caso de los hogares con un piso de mala calidad que presenta $55,9 \%$ de hogares que también son excluidos en el consumo; además el $40 \%$ de estos hogares son excluidos de la tenencia formal de la vivienda, y $44,1 \%$ son excluidos de la cobertura médica.

Sin embargo, para obtener resultados más concluyentes es necesario profundizar en futuros trabajos en los aspectos territoriales de los dos fenómenos y cómo esto afecta un desarrollo urbano homogéneo en toda la ciudad. También en las circunstancias políticas que afectan y/o determinan la incidencia tanto de la pobreza como de la exclusión en este ámbito urbano intermedio.

\section{Referencias}

Alkire, S., \& Santos, M. E. (2010). Acuate Multidimensional Poverty: A New Index for Developing Countries (No. 2010/1l). http://dx.doi.org/10.2139/ssrn.1815243

Arriagada, I. (2005). Dimensiones de la pobreza y políticas desde una perspectiva de genero. Revista de La CEPAL, 85, 101-113. Recuperado de http://www.bvsde.paho.org/bvsacd/cd30/arriagada.pdf

Atkinson, A. B. (1987). On the Measurement of Poverty. Econometrica, 55(4), 749-764. https://doi. org/10.2307/1911028

Bhalla, A. \& Lapeyre, F. (1997). Social Exclusion: Towards an Analytical and Operational Framework. Development and Change, 28(3), 413-433. https://doi.org/10.1111/1467-7660.00049

Boltvinik, J. (2003). Conceptos y medición de la pobreza. La necesidad de ampliar la mirada. Papeles de Población, Octubre/Di(38), 9-25.

Boltvinik, J. (2013). Medición multidimensional de pobreza. América Latina de precursora a rezagada. Sociedad Y Equidad, Enero(5), 4-29.

Bossert, W., D'ambrosio, C. \& Peragine, V. (2007). Deprivation and social exclusion. Economica, 74(296), 777-803. https://doi.org/10.1111/j.1468-0335.2006.00572.x

Bradshaw, J., Williams, J., Levitas, R., Pantazis, C., Patsios, D., Townsend, P., ... Middleton, S. (2000). The relationship between poverty and social exclusion in Britain. Recuperado de https://www. researchgate.net/publication/237453650_THE_RELATIONSHIP_BETWEEN_POVERTY_AND_SOCIAL_EXCLUSION_IN_BRITAIN 
Burchardt, T., Le Grand, J. \& Piachaud, D. (1999). Social Exclusion in Britain 1991-1995. Social Policy \& Administration, 33(3), 227-244. https://doi.org/10.1111/1467-9515.00148

Calle, C., Formicella, M. M., Ibañez, M., Krüger, N., Pérez, S. M. y Santos, M. (2017). Diseño del cuestionario para la encuesta del PUE Consideraciones teóricas y metodológicas (Documento de Trabajo Proyecto Unidad Ejecutora "Inclusión social: innovaciones y políticas públicas. Un análisis regional" No. 1). Recuperado de https://www.iiess-conicet.gob.ar/images/Documentos-detrabajo-PUE/2017/1GENERAL.pdf

Calle, C., London, S. y Pérez, S. M. (2016). Migración, pobreza y segregación urbana en una ciudad intermedia como Bahía Blanca. Revista Conflicto Social, 9(16), 34-59. Recuperado de http:// publicaciones.sociales.uba.ar/index.php/CS

Castell, R. (1997). La metamorfosis de la cuestión social. Buenos Aires, Argentina: Editoral Piaódos.

Centro Regional de Estudios Económicos de Bahía Blanca Argentina. (2014a). Estimación del producto bruto del partido de Bahía Blanca. Recuperado de http://www.creebba.org.ar/main/index. php?op=pbi\&cual=2014

Centro Regional de Estudios Económicos de Bahía Blanca Argentina. (2014b). Pobreza en Bahía Blanca. Recuperado de http://www.creebba.org.ar/iae/iael34/Pobreza_en_Bahia_Blanca_IAE_134.pdf

Centro Regional de Estudios Económicos de Bahía Blanca Argentina. (2017). Indicadores de Actividad. Recuperado de http://www.creebba.org.ar/main/index.php?op=charts\&cap=2

Chakravarty, S. R. \& D'Ambrosio, C. (2006). The Measurement of Social Exclusion. Review of Income and Wealth, 52(3), 377-398.

Commission Communities of European. (1992). Towards a Europe of Solidarity. Luxemburgo, Luxemburgo: European Commission.

Daly, M. \& Silver, H. (2008). Social Exclusion and Social Capital : A Comparison and Critique. Theory and Society, 37(6), 537-566.

De Haan, A. (2000). Social Exclusion : Enriching the Understanding of Deprivation. Studies in Social and Political Thought, 2(2), 22-40.

Domínguez, J. y Martín, A. M. (2006). Medición de la pobreza : una revisión de los principales indicadores. Revista de Métodos Cuantitativos Para La Economía Y La Empresa, I(2), 27-66. Recuperado de http://www.upo.es/RevMetCuant/art5.pdf

Faiguenbaum, S.(2013) Características y evolución dela pobreza,la desigualdad y las políticas en zonas rurales de América Latina. En S. Faiguenbaum, C. Ortega y F. Soto (Eds.), Pobreza rural y políticas públicas en América Latina y el Caribe (pp. 9-15). Santiago, Chile: Organizaciones de las Naciones Unidas para la Alimentación y la Agricultura.

Feres, J. C. y Mancero, X. (2001). Enfoque para la medición de la pobreza. Breve revisión de la literatura (Estudios Estadisticos y Prospectivos No.4). Recuperado de http://www.cepal.org/publicaciones/ $\mathrm{xml} / 8 / 14038 / \mathrm{lc} 2024 \mathrm{e} . \mathrm{pdf}$ 
Foster, J. (1998). Absolute versus Relative Poverty. The American Economic Review, 88(2), 335-341. Retrieved from http://www.jstor.org/stable/116944

Foster, J. E., Greer, J. \& Thorbecke, E. (1984). A Class of Decomposable Poverty Measures. Econometrica, 52(3), 761-766. https://doi.org/10.2307/1913475

Gallego, L. M. (2009). Acercamiento al problema social de la pobreza de las nociones de pobreza y los mecanismos causales. Revista Trabajo Social, (9), 1-29.

Hernández, M. (2010). El estudio de la pobreza y la exclusión social. Aproximación cuantitativa y cualitativa. Revista Interuniversitaria de Formación Del Profesorado, 69(24,3), $25-46$.

Klasen, S. (1998). Social Exclusion and Children in OECD Countries: some conceptual issues. Center for Educational Research and Innovation, OECD. Retrieved from http://www.oecd.org/edu/ school/1856923.pdf

Laderchi, C., Saith, R. \& Stewart, F. (2003). Does it matter that we don't agree on the definition of poverty? A comparison of four approaches (No. 107). QEH Working Paper Series. https://doi. org/10.1080/1360081032000111698

Laparra, M., Obradors, A., Pérez, B., Pérez, M., Renes, V., Sarasa, S., ... Trujillo, M. (2007). Una propuesta de consenso sobre el concepto de exclusión. Implicaciones metodológicas. Revista Española Del Tercer Sector, 5(enero-abril), 15-57. Recuperado de https://dialnet.unirioja.es/servlet/ articulo? codigo $=2376685$

Levitas, R. (2006). The Concept and Measurement of Social Exclusion. In C. Pantazis, D. Gordon, \& R. Levitas (Eds.), Poverty and Social Exclusion in Britain (pp. 123-160). Bristol, UK: Policy Press.

Morales-Ramos, M. A. y Morales-Ramos, E. (2008). La teoría de conjuntos difusos como una opción para medir la pobreza: El caso de México. El Trimestre Económico, 75(299), $641-662$.

Navarro, S. y Larrubia, R. (2006). Indicadores para medir situaciones de vulnerabilidad social. Propuesta realizada en el marco de un proyecto Europeo. Baetica, Estudios de Arte, Geografía E Historia, (28), 485-506.

Peralta, A., García, J. A. y Johnson, N. (2006). Dinámica y definición de pobreza en los Andes colombianos: enfoques participativos versus enfoques objetivos. Revista Desarrollo Y Sociedad, 2(58), 209243. Recuperado de http://economia.uniandes.edu.co/investigaciones_y_publicaciones/CEDE/ Publicaciones/Revista_Desarrollo_y_Sociedad/Ediciones/revista_desarrollo_y_sociedad_no_58/ dinamica_y_definicion_de_pobreza_en_los_andes_colombianos_enfoques_participativos_versus_enfoque

Preiss, O., Gorenstein, S., Hernández, J., Landriscini, G., Napal, M., Urriza, G. y Olea, M. (2012). Principales discusiones en torno a la dinámica de las ciudades intermedias. En Economía Urbana y ciudades intermedias: trayectorias pampeanas y norpatagónicas (pp. 19-45) Buenos Aires, Argentina: Ciccus.

Raya-Díez, E. (2007). Exclusión social: indicadores para su estudio y aplicación para el trabajo social. Revista Del Ministerio de Trabajo E Inmigración, (70), 155-172. Recuperado de http://www.empleo. gob.es/es/publica/pub_electronicas/destacadas/revista/numeros/70/Infol.pdf 
Santos, M. E. (2016). Pobreza por Ingresos en Argentina y Bahía Blanca: Estimaciones de referencia y cuestiones metodológicas. Actualidad Económica, (89), 5-27. Recuperado de https://revistas.unc. edu.ar/index.php/acteconomica/article/view/15750/15538

Sen, A. (2000). El desarrollo como libertad. Gaceta Ecológica, 55, 14-20. Recuperado de http://www. redalyc.org/html/539/53905501/

Spicker, P. (2007). Definitions of poverty: twelve clusters of meaning. In P. Spicker, S. Alvarez \& D. Gordon (Eds.), Poverty: An International Glossary (pp. 229-243). London, UK: Zed.

Subirats, J. (2005). ¿Es el territorio urbano una variable significativa en los procesos de exclusión e inclusión social? X Congreso del CLAD sobre la reforma del Estado y la Administración pública. https://doi.org/10.1017/CBO9781107415324.004

Touraine, A. (1991). Face à l'exclusion. Esprit, (février), 7-13. https://doi.org/10.2307/24275018 
Anexo

Anexo 1. Variables utilizadas como indicadores sintomáticos de pobreza

\begin{tabular}{|c|c|c|c|c|c|}
\hline Dimensión & \multicolumn{2}{|c|}{ Indicador Sintomático de Pobreza } & \multirow{2}{*}{$\begin{array}{c}\text { Valor } \boldsymbol{y}_{\boldsymbol{j}} \\
1\end{array}$} & \multirow{2}{*}{$\frac{\boldsymbol{\mu}_{\boldsymbol{A}}}{1}$} & \multirow{2}{*}{$\begin{array}{c}\begin{array}{c}\text { Porcentaje en la } \\
\text { población }\end{array} \\
0,62 \%\end{array}$} \\
\hline & Sabe leer y & No & & & \\
\hline & escribir & $\mathrm{Si}$ & 2 & 0 & $99,38 \%$ \\
\hline & \multirow{7}{*}{ Nivel educativo } & Sin instrucción & 0 & 1 & $2,85 \%$ \\
\hline & & Primaria incompleta & 1 & 1 & $6,21 \%$ \\
\hline & & Primaria completa & 2 & 0,6 & $23,60 \%$ \\
\hline & & Secundaria incompleta & 3 & 0,3 & $14,04 \%$ \\
\hline & & Secundaria completa & 4 & 0 & $26,86 \%$ \\
\hline & & Universitario incompleto & 5 & 0 & $6,10 \%$ \\
\hline & & $\begin{array}{l}\text { Universitario } \\
\text { completo }\end{array}$ & 6 & 0 & $20,35 \%$ \\
\hline & \multirow{3}{*}{ Material del piso } & Tierra & 1 & 1 & $0,07 \%$ \\
\hline & & Cemento & 2 & 0,5 & $2,98 \%$ \\
\hline & & Baldosa & 3 & 0 & $96,95 \%$ \\
\hline & \multirow{6}{*}{ Material del techo } & Plástico & 1 & 1 & $0,14 \%$ \\
\hline & & Chapa de metal & 2 & 0,6 & $41,8 \%$ \\
\hline & & Teja & 3 & 0,3 & $12,21 \%$ \\
\hline & & Losa sin cubierta & 4 & 0 & $11,71 \%$ \\
\hline & & Cubierta asfáltica & 5 & 0 & $18,5 \%$ \\
\hline & & Propiedad horizontal & 5 & 0 & $15,5 \%$ \\
\hline & \multirow{3}{*}{ Desagüe } & Pozo ciego & 1 & 1 & $4,25 \%$ \\
\hline & & Cámara séptica & 2 & 0,5 & $17,3 \%$ \\
\hline & & Cloaca & 3 & 2 & $78,3 \%$ \\
\hline & \multirow{3}{*}{$\begin{array}{l}\text { Ingreso familiar } \\
\text { total per cápita* }\end{array}$} & $\begin{array}{l}\text { Ingreso inferior al } 60 \% \text { de } \\
\text { la media }\end{array}$ & $Y<=\$ 2841$ & 1 & $31,72 \%$ \\
\hline & & $\begin{array}{l}\text { Ingreso entre el } 60 \% \text { de la } \\
\text { media y la media }\end{array}$ & $\$ 2841<Y<=\$ 4735$ & $\frac{(4735-Y)}{1894}$ & $38,46 \%$ \\
\hline & & Ingreso mayor & $Y>4735 \$$ & 0 & $39,82 \%$ \\
\hline
\end{tabular}

* Se elige el 60\% de ingreso medio como la línea de pobreza relativa elegida para la Unión Europea.

Fuente: elaboración propia. 